SHOCK WAVE TRANSIT THROUGH THE TRANSITION REGION AND RELATED RADIO FLUCTUATIONS

Giannina Poletto

Osservatorio Astrofisico di Arcetri, Firenze, Italy

ABSTRACT - The perturbing effects of a shock wave transit through the transition region are evaluated, using a simplified method of solution of the system of partial differential equations describing the fluid motion behind the shock passage. Calculated temperatures and densities are given at successive times during the shock transit. Based on these shocked models, time dependent brightness temperatures are derived at several decimetric wavelengths and compared with the unperturbed values to evaluate the amplitude of the induced brightness fluctuations. Finally, the possibility of detecting such shock induced phenomena is discussed.

\title{
INTRODUCTION
}

In the last decade a number of authors have made observations of the solar radio emission attempting to detect the fluctuations whose presence at optical wavelengths has been established for a long time (Leighton et al. 1962). Unfortunately, radio measurements lead to contradictory results due to both the low resolving power of radio instruments and the atmospheric fluctuations, which have periods similar to those expected from the solar data.

In spite of these difficulties, substantial evidence has been accumulated indicating the presence of fluctuations with periods ranging from tens of seconds to tens of minutes from millimetric up to decimetric wavelengths (see, for instance, Butz et al., 1979, and references therewith enclosed).

Theoretically, at centimetric wavelengths, the possibility that radio fluctuations could be ascribed to large scale solar oscillations has been investigated by Shuter (1975). Alternatively, Lang (1974), and Butz et al. (1979), claimed their experimental results were consistent with those derived from the hypothesis of acoustic waves propagating upwards through the solar atmosphere.

The former interpretation seems to be discarded due to the variability of the radio fluctuations with respect to the high stability of

M.R. Kundu and T.E. Gergely (eds.), Radio Physics of the Sun, 57-60.

Copyright $\odot 1980$ by the IAU. 
the global solar oscillations. If the latter interpretation is correct, the fluctuations at decimetric wavelengths would have to be induced by shock waves, since acoustic waves at the centimetric emission levels are expected to steepen into shocks at the levels responsible for the decimetric emission. In the following sections, we give the equations which allow us to calculate the temperature and density perturbations caused by the shock transit. The resulting brightness temperatures are then computed and compared with their unperturbed values to determine the amplitude of the induced brightness fluctuations, and their observational detectability.

\section{THE METHOD}

To evaluate how the physical parameters are affected by the passage of the shock wave, we have assumed that a shock is already present at the base of the transition region and is propagating vertically up to the coronal layers, through an atmosphere with no mass motions and with negligible or only vertical magnetic fields. Possible wave periodicities are not considered, which is valid as long as interaction effects between successive shocks are negligible.

The equations describing the fluid motion behind the shock front during a limited time interval $\Delta t$, shorter than the radiative and conductive cooling times, are

$$
\left\{\begin{array}{l}
\frac{\partial v}{\partial t}+v \frac{\partial v}{\partial h}+\frac{1}{\rho} \frac{\partial p}{\partial h}=-g \\
\frac{\partial \rho}{\partial t}+\rho \frac{\partial v}{\partial h}+v \frac{\partial \rho}{\partial h}=0 \\
\frac{\partial s}{\partial t}+v \frac{\partial s}{\partial h}=0
\end{array}\right.
$$

where symbols have their usual meaning ( $s$ is the specific entropy) and the temperature and density jumps at the shock discontinuity are given by the Rankine Hugoniot relations.

The method used to solve the preceding set of partial differential equations is fully described elsewhere (Poletto, 1979). Perturbed models of temperature and density at times $t<\Delta t$ during the shock transit, allow us to evaluate the shock perturbed brightness temperatures $T_{b}, v$
at frequency $v$, through the relation

$$
\mathrm{T}_{\mathrm{b}, \nu}=\int \mathrm{T} \mathrm{e}^{-\tau \nu} \mathrm{d} \tau_{\nu}
$$

where the optical depth is given by

$$
\tau_{\nu}=\frac{\xi}{\nu^{2}} \int \frac{\mathrm{N}^{2} \mathrm{dh}}{\mathrm{T}^{3 / 2}\left(1-\mathrm{N} / \mathrm{N}_{\mathrm{o}}\right)^{\frac{1}{2}}}
$$

and $\mathrm{N}=\mathrm{N}(\mathrm{h}), \mathrm{T}=\mathrm{T}(\mathrm{h})$ are the shock modified temperature and density, $\mathrm{N}_{\mathrm{O}}$ is the critical density, and $\xi$ is a constant. 


\section{THE RESULTS}

Applying the preceding procedure, the relative increase $\Delta \mathrm{T}_{\mathrm{b}}$ of the perturbed brightness temperatures $\mathrm{T}_{\mathrm{b}}$, Per over the unperturbed ones $\mathrm{T}_{\mathrm{b}}$, Unp have been computed for $\lambda=60,80,100 \mathrm{~cm}$ at several times after the shock passage. The results and the percentage values $\Delta \mathrm{T}_{\mathrm{b}} / \mathrm{T}_{\mathrm{b}}$, Unp are illustrated in the following Table

\begin{tabular}{|c|c|c|c|c|}
\hline$\lambda(\mathrm{cm})$ & $\Delta \mathrm{T}_{\mathrm{b}} \quad(\mathrm{t}=10 \mathrm{~s})$ & $\Delta \mathrm{T}_{\mathrm{b}} \quad(\mathrm{t}=20 \mathrm{~s})$ & $\Delta \mathrm{T}_{\mathrm{b}}$ & $(t=30 \mathrm{~s})$ \\
\hline 60 & $44140 \quad(10 \%)$ & - & & - \\
\hline 80 & 13940 & 13140 & & - \\
\hline 100 & $490(0.1 \%)$ & $4240(0.5 \%)$ & 37060 & $(5 \%)$ \\
\hline
\end{tabular}

The observability of these brightness fluctuations depends on the size and number of fluctuating elements, and on the resolving power of the observing instrument. Assuming a Poisson distribution for the number of the oscillating elements with respect to their average value, and an angular resolution of $1 / 30$ of the solar disk, the measured temperature fluctuation will be about $1 \%$, and should be detectable.

Since the measured brightness fluctuations are of the same order of magnitude as those resulting from the present computations, a new piece of evidence in favor of the wave hypothesis has been derived. However, calculations extending to times $t>\Delta t$, as well as more observations at decimetric wavelengths, up to now very scanty, are needed before drawing definite conclusions.

\section{REFERENCES}

Butz, M., Hirth, W., and Fürst, E.: 1979, Astron. Astrophys. 72, 211-214. Lang, K.R.: 1974, Astrophys. J. 192, 777-786.

Leighton, R.B., Noyes, R.W., and Simon, G.W.: 1962, Astrophys. J. 135, 474-499.

Poletto, G.: 1979, Solar Phys. 61, 389-405.

\section{DISCUSSION}

Alissandrakis: I wish to point out that there is serious doubt about the oscillatory nature of the fluctuations at $\mathrm{cm}$ wavelengths. Kundu and myself, among others, using the NRAO interferometer at 3.7 and $11.1 \mathrm{~cm}$ did not find any oscillatory components, although we could clearly see the fluctuations. 
Poletto: I know that different interpretations of the experimental results have been given and that Kundu and Alissandrakis (1975) could not confirm some of the preceeding results.

I think that radio observers can comment on this problem better than I; my feeling is that evidence is accumulating in favor of the real presence of oscillations in the solar atmosphere. For instance, their existence at decimetric wavelengths - which I have just discussed - has been confirmed in recent papers by Abranin et al. (1978), and Snegrive (1979).

As far as the present results are concerned, the periodic or aperiodic character of the fluctuations does not affect them, as I considered the effects of a single shock pulse, and I made no hypothesis about the periodic or aperiodic occurrence of shocks.

Dulk: Would the presence of your postulated shock waves contribute a significant amount to the observed brightness temperature at any wavelength?

Poletto: The amplitude of the brightness temperature fluctuations which would be measured by an instrument depend on several factors, such as its spatial resolution and the size and number of elements fluctuating at any given moment. If we assume that oscillatings elements, distributed all over the Sun, have diameters of some ten thousands of kilometers (Butz et al., 1979) and that about $2 / 3$ of the solar surface is experiencing oscillations at any moment (Stein and Leibacher, 1974), an instrument capable to resolve $1 / 30$ of the solar disk, will measure brightness temperature fluctuations of the order of $1 \%$. 